\title{
IGOST aktuell
}

\section{Klausurtagung in Oberstdorf als Aufgalopp ins neue Jahr}

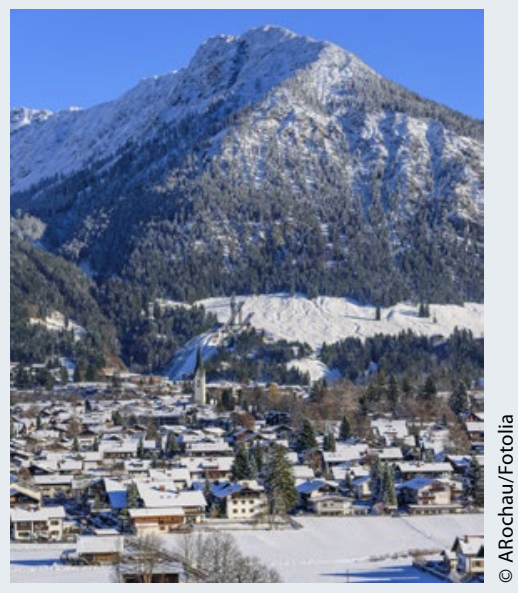

Im Januar 2017 startet das Jahr für die IGOST mit der traditionellen Klausurtagung in Oberstdorf. Vorstandsmitglieder, Senatoren, Beiräte und eingeladene Gäste beschäftigen sich mit den internen Themen der Fachgesellschaft ebenso wie mit der Weiterentwicklung der Schmerztherapie an sich.

Rückblick, Ausblick und Neuausrichtung, Bewertung der im vergangenen Jahr stattgefundenen Veranstaltungen gehören hier ebenso zum Themenspektrum wie die Planung kommender Aktivitäten. Personell sind die traditionell immer gut nachgefragten Seminarangebote, wie beispielsweise die Kurse zum Erlernen der wirbelsäulennahen Injektionen zu besetzen; gleiches gilt für neue Angebote, zum Beispiel für die DWG. Zunehmend sind auch Personalressourcen erforderlich für die Beteiligung an der Erstellung von AWMF-Leitlinien, beispielsweise zu den Themen zervikale und lumbale Radikulopathie, CRPS und weitere.

Letztlich lassen sich die zahlreichen und vielfältigen Aufgaben, die wir uns für 2017 vorgenommen haben, nur mit einem hoch motivierten und mit großer Freude arbeitenden Team erfolgreich bewältigen. Somit hoffen wir, wie alle Jahre wieder, auch zu Beginn des noch neuen Jahres auf den Beistand des "Geists von Oberstdorf".

\section{Termine}

Hands-on-Kurse

Injektionstechniken Wirbelsäule 1.7.2017 in Mülheim

Kursreihe Botulinumtoxin

Grundkurs

12. und 13.5.2017 in Köln

Spezialkurs

30.6. und 1.7.2017 in Köln

Sitzungen im Rahmen

der Frühjahrstagung der VSOU

in Baden Baden

27.4.2017

- Schmerztherapie bei Osteoporose

- Seminar Begutachtung in O\&U

- Schmerztherapie - Update

- Einsatz von Kortikoiden an der Wirbelsäule

\subsubsection{7}

- ordentliche Mitgliederversammlung der IGOST e. V.

- Update CRPS

- Implikation des §299a für die Klinik und Praxis in O\&U

\subsubsection{7}

- Abrechnungsseminar O\&U

- Workshop Botulinumtoxin in O\&U

- Injektionskurs Gelenke

- Re-Zertifizierungskurs für das IGOST

Zertifikat "Ambulante/stationäre

Schmerztherapie"

Infos und Anmeldung

IGOST-Koordinationszentrum

Grüner-Turm-Str. 4-10

88212 Ravensburg

E-Mail: post@igost.de

Fax: 0751/35559797

Internet: www.IGOST.de

\section{IGOST-Aktivitäten rund um Leitlinien}

\section{Sehr geehrte Kolleginnen, sehr geehrte Kollegen,}

die IGOST arbeitet als Vertreterin der DGOOC und darüber hinaus durch die Senatoren und Vorstandsmitglieder der IGOST als Vertreter anderer Fachgesellschaften an der Erstellung und Überarbeitung zahlreicher Leitlinien aktiv mit. Hier eine kurze Übersicht:

_spezifischer Kreuzschmerz (S2k): M. Strohmeier, H. Locher für die DGM

_Schmerzassessment bei älteren Menschen in der vollstationären Altenhilfe (S3): C. Schott

_CRPS (S1): S. Middeldorf

_Schmerzmanagement bei Querschnittslähmung (S2k): C. Schott

_ Gonarthrose (S2k): F. Bock, J. Jerosch

_Coxarthrose (S2k): F. Bock

_NVL nicht spezifischer Kreuzschmerz (S3): F. Bock, HRC für die DGSS, H. Locher für die DGM

_zervikale Radikulopathie (S1): H. Locher für die DGM

_ Brustschmerz (S2): H. Locher für die DGM

_axiale Spondylarthritis (S3): H. Locher für die DGM

Bei Fragen zu den einzelnen Leitlinien stehen wir Ihnen gerne zur Verfügung. Bitte senden Sie uns eine E-Mail an post@igost.de. 